\title{
Population pharmacokinetics of nintedanib, an inhibitor of tyrosine kinases, in patients with non-small cell lung cancer or idiopathic pulmonary fibrosis
}

\author{
Ulrike Schmid $^{1} \cdot$ Karl-Heinz Liesenfeld $^{1} \cdot$ Angele Fleury $^{1} \cdot$ Claudia Dallinger $^{1}$ • \\ Matthias Freiwald ${ }^{1}$
}

Received: 20 June 2017 / Accepted: 10 October 2017 / Published online: 8 November 2017

(C) The Author(s) 2017. This article is an open access publication

\begin{abstract}
Purpose A population pharmacokinetic model was developed for nintedanib in patients with non-small cell lung cancer (NSCLC) or idiopathic pulmonary fibrosis (IPF). The effects of intrinsic and extrinsic patient factors on exposure of nintedanib and its main metabolite BIBF 1202 were studied.

Methods Data from 1191 patients with NSCLC $(n=849)$ or IPF $(n=342)$ treated with oral nintedanib (once- or twice-daily, dose range $50-250 \mathrm{mg}$ ) in 4 Phase II or III studies were combined. Plasma concentrations of nintedanib $(n=5611)$ and BIBF $1202(n=5376)$ were analyzed using non-linear mixed-effects modeling.

Results Pharmacokinetics of nintedanib were described by a one-compartment model with linear elimination, first-order absorption, and absorption lag time. For a typical patient, the absorption rate was $0.0827 \mathrm{~h}^{-1}$, apparent total clearance was $897 \mathrm{~L} / \mathrm{h}$, apparent volume of distribution at steady state was $465 \mathrm{~L}$, and lag time was $25 \mathrm{~min}$. Age, weight, smoking, and Asian race were statistically significant covariates influencing nintedanib exposure, but no individual covariate at extreme values (5th and 95th percentiles of baseline values for continuous covariates) resulted in a change of more than $33 \%$ relative to a typical patient. Pharmacokinetics and
\end{abstract}

Electronic supplementary material The online version of this article (doi:10.1007/s00280-017-3452-0) contains supplementary material, which is available to authorized users.

Ulrike Schmid

ulrike_1.schmid@boehringer-ingelheim.com

1 Department of Translational Medicine and Clinical Pharmacology, Boehringer Ingelheim Pharma GmbH \& Co. KG, Birkendorfer Strasse 65, 88397 Biberach an der Riss, Germany covariate effects for BIBF 1202 were similar to nintedanib. Mild or moderate renal impairment and mild hepatic impairment (classified by transaminase or bilirubin increase above the upper limit of normal) or underlying disease had no significant effects on nintedanib pharmacokinetics.

Conclusions This model adequately described the pharmacokinetic profile of nintedanib in NSCLC and IPF populations and can be used for simulations exploring covariate effects and exposure-response analyses.

Keywords Covariates - Idiopathic pulmonary fibrosis . Nintedanib · Non-small cell lung cancer · Population pharmacokinetics

\section{Introduction}

Nintedanib is a kinase inhibitor targeting VEGFR (vascular endothelial growth factor receptor) 1,2 , and 3, PDGFR (platelet-derived growth factor receptor) $\alpha$ and $\beta$, and FGFR (fibroblast growth factor receptor) 1, 2, and 3 [1-3]. These kinase receptors have been shown to play an important role not only in angiogenesis but also in tumor growth and metastasis [4]. Nintedanib has shown clinical efficacy in non-small cell lung cancer (NSCLC) [5, 6] and in 2014 was approved in Europe in combination with docetaxel for use in adult patients with locally advanced, metastatic, or locally recurrent NSCLC of adenocarcinoma tumor histology, after firstline chemotherapy [3, 7].

VEGFR, PDGFR, and FGFR have also been shown to participate in the mechanisms underlying the disease of idiopathic pulmonary fibrosis (IPF) [8-10]. Therefore, the specific kinase inhibitory profile of nintedanib was considered to be a potentially beneficial therapy for IPF, a chronic, devastating disease of unknown etiology characterized by 
progressive fibrotic destruction of the lung. Based on results of one Phase II trial (TOMORROW) [11] and two identically designed Phase III trials (INPULSIS- 1 and INPULSIS-2), which demonstrated a significant reduction in the rate of decline in forced vital capacity over a 52-week treatment period [12], nintedanib is approved for the treatment of IPF in various countries, including the US, Canada, Japan, and Europe [13, 14].

In patients with solid tumors, peak plasma concentrations of nintedanib occur approximately $2-4 \mathrm{~h}$ after oral dosing [15-17]. Nintedanib is typically administered in twice-daily doses, with steady state achieved within 7 days of twicedaily dosing $[18,19]$. The total and peak systemic exposures are dose-proportional and absolute bioavailability of nintedanib is about $5 \%$ [19]. The total fraction of nintedanib and its metabolites absorbed is estimated to be much higher than the absolute bioavailability of nintedanib alone (23 versus $5 \%$, [19]), suggesting a high intestinal and/or hepatic firstpass metabolism. There is moderate-to-high inter-subject variability for the area under the plasma concentration-time curve for the dosing interval at steady state $\left(\mathrm{AUC}_{\tau, \mathrm{ss}}\right)[15,16$, 20], and the geometric mean terminal elimination half-life is about 10-15 h [16, 19]. In a Phase I trial, food increased nintedanib exposure $\left(\mathrm{AUC}_{0-\infty}\right)$ and maximum plasma concentrations $\left(C_{\max }\right)$ by $20 \%$ and absorption was delayed [17]. In Phase II and III trials, nintedanib was generally administered with food and current prescribing guidelines recommended that it should be taken with food [13].

Nintedanib is rapidly metabolised, primarily via ester cleavage, resulting in the formation of the free acid moiety (BIBF 1202) that is subsequently glucuronidated by hepatic and intestinal uridine 5'-diphospho-glucuronosyltransferase (UGT) 1A1 (UGT1A1) enzymes to form BIBF 1202 glucuronide and excreted in the feces (about $94 \%$ of the dose) [16]. Less than $1 \%$ is eliminated in urine. Drug interactions based on cytochrome P-450 pathways are not expected. Nintedanib is a substrate of P-glycoprotein (P-gp) and co-administration of potent $\mathrm{P}$-gp modulators is associated with small-tomoderate changes in exposure (e.g., co-administration with $400 \mathrm{mg}$ ketoconazole [potent inhibitor], increased nintedanib exposure by $60 \%$, whereas co-administration of nintedanib with $600 \mathrm{mg}$ rifampicin [potent inducer] decreased nintedanib exposure by $50 \%[13,21,22])$.

Although the principal metabolite BIBF 1202 shows pharmacological activity at some of the target receptors, in vivo cellular activity indicates a substantially lower potency compared to nintedanib ( 9-10-fold based on VEGF or bFGF stimulation of human umbilical vascular endothelial cells; 265- and 607-fold based on PDGFR $\alpha$ and PDGFR $\beta$ stimulation of primary lung fibroblasts, respectively) (Boehringer Ingelheim unpublished data, [14]). BIBF 1202 was not effective in mouse xenograft models [7], suggesting that BIBF 1202 plasma levels themselves are unlikely to contribute to the clinical effects of nintedanib in vivo. BIBF 1202 glucuronide did not show in vitro activity at the target receptors and is a non-reactive glucuronide [16].

The primary objective of this population pharmacokinetic analysis was to characterize the pharmacokinetic plasma profile of nintedanib in the target populations of NSCLC and IPF patients and to evaluate the effect of selected intrinsic and extrinsic factors on exposure of nintedanib. In view of its lower in vivo potency compared with nintedanib, a secondary objective was to characterize plasma exposure of the metabolite BIBF 1202 and to assess the influence of intrinsic and extrinsic factors on its exposure.

\section{Subjects and methods}

\section{Trials included in the analysis}

This population pharmacokinetic analysis pooled data from nintedanib treatment in four clinical studies; 1 Phase II $(n=73)$ [23] and two Phase III trials in patients with NSCLC (LUME-Lung $1(n=652)$ [5] and LUME-Lung 2 $(n=347)$ [6]) and one Phase II trial (TOMORROW, $n=343$ ) in patients with IPF [11] (data from IPF Phase III trials were not available at time of analysis). An overview of these studies is provided in Online Resource Table S1. In the NSCLC Phase II trial [23], patients with locally advanced or metastatic relapsed NSCLC in whom first- or second line platinum-based chemotherapy had failed and were randomized to nintedanib 150 or $250 \mathrm{mg}$ twice-daily. Treatment continued until disease progression or intolerable toxicity. In the NSCLC Phase III trials, nintedanib $200 \mathrm{mg}$ twice-daily or placebo was given in repeated 21 day treatment cycles in combination with single infusions of docetaxel $75 \mathrm{mg} / \mathrm{m}^{2}$ (LUME-Lung 1 trial [5]) or pemetrexed $500 \mathrm{mg} / \mathrm{m}^{2}$ (LUMELung 2 trial [6]), administered on day 1 of each cycle. Treatment cycles were continued until disease progression or intolerable toxicity. In the Phase II trial in patients with IPF [11], patients were randomized to a 52-week treatment period with one of the four doses of nintedanib $(50 \mathrm{mg}$ once or twice-daily, 100, $150 \mathrm{mg}$ twice-daily) or placebo. In all four trials, dose reductions and interruptions were allowed to manage adverse events. All studies were conducted in accordance with the Declaration of Helsinki and with approval of the local ethics committees. Written informed consent was obtained from all patients before study entry.

\section{Pharmacokinetic sampling and bioanalytical assays}

Blood sampling schema for measurement of nintedanib plasma concentrations varied. For all trials, at least two predose and two post-dose blood samples were scheduled during treatment (see Online Resource Table S1). In addition, 
more extensive sampling was performed in the two Phase II trials $[11,23]$. Nintedanib and BIBF 1202 plasma concentrations were analyzed by validated liquid chromatography-mass spectrometry (HPLC-MS/MS) methods [16]. The calibration curves covered the range $0.5-500 \mathrm{ng} / \mathrm{mL}$ for nintedanib and 1-1000 ng/mL for BIBF 1202 in undiluted plasma samples and were linear over this range.

\section{Data analysis}

The population pharmacokinetic analysis was performed using non-linear mixed-effects modeling techniques (NONMEM, version VI 2.0, ICON Development Solutions, Ellicott City, Maryland, USA). The first-order conditional estimation algorithm with interaction was used. Data processing, diagnostic plots, and calculation of descriptive statistics were performed using R version 2.12 .1 (http://www. rproject.org) and SAS version 9.2 (SAS Institute Inc, Cary, NC, USA). Bootstrap analysis was performed using Perlspeaks-NONMEM, version 3.5.3 [24, 25].

\section{Base model development}

A sequential approach was used for population pharmacokinetic model development. First, the base model was developed for nintedanib by (1) investigating the compartmental model structure including evaluation regarding dose linearity as well as trial effects and (2) investigating the stochastic model. Inter-individual variability (IIV, $\eta$ ) and inter-occasion variability $(\mathrm{IOV}, \kappa)$ were modeled using exponential random effect models. For testing IOV, each visit was defined as one occasion. IIV, IOV, and residual (unexplained) variability $(\varepsilon)$ were assumed to be symmetrically distributed around 0 with variances $\omega_{\mathrm{IIV}}{ }^{2}, \omega_{\mathrm{IOV}}{ }^{2}$, and $\sigma^{2}$, respectively. Model selection was guided by change in objective function values (OFV), identifiability of parameters and precision of parameter estimates, correlation between the estimates of fixed-effect parameters, numerical stability, ability to obtain a successful COVARIANCE step, and visual inspection of basic goodness-of-fit plots.

Using fixed pharmacokinetic parameter estimates from the nintedanib base model (typical values plus empirical Bayes estimates of individual $\eta$ ), the base model of BIBF 1202 was developed. Plasma concentrations of nintedanib and BIBF 1202 were reported in $\mathrm{nM}$ to account for differences in their molecular weight.

\section{Covariate analysis}

The effects of intrinsic patient factors (e.g., gender, age, body weight, ethnicity, laboratory values, and NSCLC histology) and extrinsic factors (e.g., smoking history and alcohol consumption) on pharmacokinetic parameters of nintedanib were evaluated by applying a stepwise forward inclusion/backward elimination approach. Combinations of covariates and model parameters to be tested were pre-specified based on prior knowledge, physiological plausibility, or general clinical interest (see Online Resource Table S2). Potential effects of patient population (NSCLC versus IPF) or treatment regimen (monotherapy versus combination therapy with docetaxel or pemetrexed) could not be distinguished from trials effects, and were, therefore, not reevaluated during covariate analysis.

To assess the effect of hepatic impairment on apparent total plasma clearance $(\mathrm{CL} / \mathrm{F})$ and relative bioavailability (F1), alanine transaminase (ALT), aspartate transaminase (AST), and total bilirubin (BIL) were tested individually and in combination using an adapted liver dysfunction group (LDF) composite developed for organ dysfunction studies by the Organ Dysfunction Working Group of the National Cancer Institute [26-28]. In this classification, hepatic dysfunction is classified as mild (subdivided into two subgroups), moderate, or severe according to ALT, AST, and BIL levels relative to the upper limit of normal (ULN) (see Table 1). This categorization was preferred over the classical Child-Pugh classification [29] (based on assessments of BIL, albumin, prothrombin time, encephalopathy, and ascites) commonly used to assess surgical risk in cirrhotic patients, as it has potential disadvantages for use in oncology patients [30]. Creatinine clearance $\left(\mathrm{CL}_{\mathrm{CR}}\right)$ estimated by the Cockcroft-Gault equation [31] was used as surrogate for renal function. The effect of UGT1A1 polymorphisms was studied by analyzing homozygous and heterozygous genotypes separately or in combination collected in the phase II trials [11, 23].

Univariate assessment of all pre-specified covariate effects using various functions (linear, hockey stick, power, or step) was initially performed to guide further covariate model building (see Online Resource Table S2). This was followed by a forward inclusion and backward elimination procedure, with significance levels of 5 and $0.1 \%$ (log-likelihood ratio

Table 1 Definition of liver dysfunction groups based on adapted liver dysfunction group composite developed for organ dysfunction studies by the Organ Dysfunction Working Group of the National Cancer Institute [26-28]

\begin{tabular}{lll}
\hline Variable & Transaminase levels & Total bilirubin levels \\
\hline Control & AST and ALT $\leq \mathrm{ULN}$ & $\mathrm{BIL} \leq \mathrm{ULN}$ \\
Mild 1 & AST and ALT $\leq 2.5 \times \mathrm{ULN}$ & $\mathrm{BIL} \leq \mathrm{ULN}$ \\
Mild 2 & AST and ALT $\leq 10 \times \mathrm{ULN}$ & $\mathrm{BIL} \leq 1.5 \times \mathrm{ULN}$ \\
Moderate & AST and ALT $\leq 10 \times \mathrm{ULN}$ & $1.5 \times \mathrm{ULN}<\mathrm{BIL} \leq 3 \times \mathrm{ULN}$ \\
Severe & AST or ALT $>10 \times \mathrm{ULN}$ & $\mathrm{BIL}>3 \times \mathrm{ULN}$ \\
& or & \\
\hline
\end{tabular}

$A L T$ alanine transaminase, $A S T$ aspartate transaminase, $B I L$ bilirubin, $U L N$ upper limit of normal 
test, Chi square distribution), respectively. Multiplicative covariate regression models were used to evaluate covariate combinations.

The covariate analysis for BIBF 1202 was based on the fixed pharmacokinetic parameters of the final nintedanib model and base model of BIBF 1202 and followed the same principles described for nintedanib (see Online Resource Table S3).

\section{Final model evaluation}

The predictive performance of the base and final nintedanib models was assessed by a prediction-corrected visual predictive check (pcVPC) and prediction-corrected quantitative predictive check (pcQPC), respectively. For each, 1000 data sets were simulated using the respective nintedanib model and its parameter estimates (fixed and random effects). For each simulated data set, the same number of patients, dosing history, number of observations, sampling schedule, and covariate values as in the original data were used. Observed and simulated values were prediction-corrected using the technique of Bergstrand and colleagues [32] and were compared graphically and numerically. The final nintedanib model was further evaluated by non-parametric bootstrap analysis, in which the model was fitted to 2000 bootstrap replicates generated by resampling from the original analysis data set.

Model evaluation for BIBF 1202 was analogous to nintedanib, with the exception of the non-parametric bootstrap analysis.

Finally, after the sequential pharmacokinetic analysis for nintedanib and BIBF 1202, the parameters for the final models of nintedanib and BIBF 1202 were estimated simultaneously.

\section{Simulations}

To illustrate individual covariate effects, the change in the median steady-state nintedanib and BIBF 1202 plasma concentration-time profiles were compared to the exposure in a typical patient. The typical patient was defined by baseline medians (continuous covariate) and modes (categorical covariate) of the respective covariates in the total analyzed population.

\section{Results}

\section{Description of data set}

The pharmacokinetic analysis data set comprised 1191 patients (849 NSCLC, 342 IPF) from four studies providing 5611 and 5376 nintedanib and BIBF 1202 plasma concentrations, respectively, for model development. The baseline demographic data of the patients and descriptive statistics of the tested intrinsic and extrinsic covariates are given in Table 2.

\section{Final pharmacokinetic model for nintedanib}

The pharmacokinetic profile of nintedanib was adequately described by a one-compartment model with first-order absorption and linear elimination. Inclusion of an absorption lag time (ALAG) was also required. The residual variability was based on log-transformed nintedanib plasma concentrations with an additive random effect model. IIV could be implemented in the nintedanib apparent volume of distribution $\left(\mathrm{V}_{2} / \mathrm{F}\right)$, relative bioavailability $(\mathrm{F} 1)$, and absorption-rate constant $\left(k_{\mathrm{a}}\right)$. Differences in nintedanib exposure and absorption among individual trials could not be explained by any of the investigated covariates or patient characteristics and were accounted for separately. For example, F1 was 30\% higher in the NSCLC phase II [23] and LUME-Lung 2 [6] trials than in the LUME-Lung 1 [5] and IPF phase II [11] trials; $k_{\mathrm{a}}$ was $120 \%$ higher in the phase II NSCLC and IPF trials $[11,24]$ than in the two NSCLC Phase III trials $[5,6]$. No major deviation from dose linearity was observed.

Age, body weight, ethnic origin, and smoking status were statistically significant covariates influencing nintedanib exposure (See Online Resource Table S6). F1 was affected by age and smoking status, and differed significantly between the investigated Asian subgroups. CL/F was significantly influenced by body weight.

Gender, patient population (NSCLC versus IPF; see also trial effects), NSCLC histology (adenocarcinoma versus non-adenocarcinoma), therapy regimen (monotherapy versus combination therapy with docetaxel or pemetrexed; see also trial effects), ECOG performance status, presence of liver metastases, mild or moderate renal impairment (based on $\mathrm{CL}_{\mathrm{CR}}$ ), and lactate dehydrogenase (LDH) levels had no statistically significant impact on nintedanib pharmacokinetics. Despite limited data, there was also no substantial difference in nintedanib pharmacokinetics between Black patients (9 of the 1191 patients included), alcohol consumption (11 patients included) or UGT1A1 polymorphism status.

In patients with mild hepatic impairment at start of treatment $(n=116)$, there was a statistically non-significant trend towards lower $\mathrm{CL} / \mathrm{F}$ or higher F1 values. During univariate assessment, F1 was estimated to increase by 8 and $13 \%$ for the mild $1(n=104)$ and mild $2(n=12)$ liver dysfunction categories, respectively. The limited number of patients did not allow assessment of the moderate $(n=1)$ or severe $(n=0)$ liver dysfunction categories.

Table 3 displays the final model parameter estimates for nintedanib and their precision. For a typical patient defined by the mode/median of the baseline covariate values (i.e., Caucasian, aged 62 years, weighing $71.5 \mathrm{~kg}$, ex- or 
Table 2 Summary of baseline characteristics of trial subjects
Patient characteristics

\begin{tabular}{|c|c|}
\hline No. patients & 1191 \\
\hline Age (year) & $62.0(45.0-76.0)$ \\
\hline Weight (kg) & $71.5(50.0-100.0)$ \\
\hline Female, $n(\%)$ & $367(30.8)$ \\
\hline \multicolumn{2}{|l|}{ Ethnic origin, $n(\%)$} \\
\hline Caucasian & $899(75.5)$ \\
\hline $\operatorname{Asian}^{\mathrm{a}}$ & $283(23.7)$ \\
\hline Black & $9(0.8)$ \\
\hline \multicolumn{2}{|l|}{ Smoking, $n(\%)$} \\
\hline Non-smoker & $327(27.5)$ \\
\hline Ex-smoker & $688(57.8)$ \\
\hline Current smoker & $176(14.8)$ \\
\hline \multicolumn{2}{|l|}{ Alcohol consumption, $n(\%)$} \\
\hline No alcohol & $701(58.9)$ \\
\hline Alcohol consumption should not interfere with trial participation & $479(40.2)$ \\
\hline Alcohol consumption could interfere with trial participation & $11(0.9)$ \\
\hline $\mathrm{CL}_{\mathrm{CR}}(\mathrm{mL} / \mathrm{min})^{\mathrm{b}}$ & $80.8(47.1-134.3)$ \\
\hline Alanine transaminase (U/L) & $19.0(8.0-47.0)$ \\
\hline Aspartate transaminase (U/L) & $21.1(11.5-42.0)$ \\
\hline Lactate dehydrogenase (U/L) & $238.0(141.0-576.3)$ \\
\hline Total bilirubin $(\mu \mathrm{mol} / \mathrm{L})$ & $8.2(3.4-15.6)$ \\
\hline Total protein $(\mathrm{g} / \mathrm{L})$ & $74.0(64.0-86.0)$ \\
\hline \multicolumn{2}{|l|}{ Categorization of liver dysfunction, $n(\%)$} \\
\hline Control & $1074(90.2)$ \\
\hline Mild 1 & $104(8.7)$ \\
\hline Mild 2 & $12(1.0)$ \\
\hline Moderate & $1(0.1)$ \\
\hline Severe & $0(0.0)$ \\
\hline \multicolumn{2}{|l|}{ ECOG performance score, $n(\%)$} \\
\hline 0 & $269(22.6)$ \\
\hline 1 & $562(47.2)$ \\
\hline 2 & $18(1.5)$ \\
\hline \multicolumn{2}{|l|}{ Variable } \\
\hline Missing (due to IPF indication) & $342(28.7)$ \\
\hline \multicolumn{2}{|l|}{ Indication, $n(\%)$} \\
\hline NSCLC & $849(71.3)$ \\
\hline IPF & $342(28.7)$ \\
\hline \multicolumn{2}{|l|}{ Cancer histology, $n(\%)$} \\
\hline NSCLC—no adenocarcinoma & $274(23.0)$ \\
\hline NSCLC_-adenocarcinoma & $502(42.1)$ \\
\hline Patients with IPF or NSCLC of unknown histology & $415(34.8)$ \\
\hline \multicolumn{2}{|l|}{ UGT1A1 polymorphism status ${ }^{\mathrm{c}}, n(\%)$} \\
\hline \multicolumn{2}{|l|}{ UGT1A1*27 } \\
\hline Wild-type & $198(16.6)$ \\
\hline Mutation & $0(0.0)$ \\
\hline \multicolumn{2}{|l|}{ UGT1A $1 * 60$} \\
\hline Wild-type & $62(5.2)$ \\
\hline Mutation & $136(11.4)$ \\
\hline \multicolumn{2}{|l|}{$\mathrm{UGT} 1 \mathrm{~A} 1 * 6$} \\
\hline Wild-type & $185(15.5)$ \\
\hline Mutation & $13(1.1)$ \\
\hline
\end{tabular}


Table 2 (continued)

\author{
UGT1A $1 * 28 / * 36 / * 37$ \\ Wild-type \\ Mutation $^{\mathrm{d}}$ \\ Presence of liver metastases ${ }^{\mathrm{c}}, n(\%)$ \\ No presence (+ IPF patients) \\ Presence \\ $146(12.3)$ \\ $1038(87.2)$ \\ $145(12.2)$ \\ $C L_{C R}$ creatinine clearance, ECOG Eastern Cooperative Oncology Group, IPF idiopathic pulmonary fibro- \\ sis, NSCLC non-small-cell lung cancer. Results are presented as median (5th and 95th percentiles) unless \\ stated otherwise \\ ${ }^{a}$ Asian patients included Chinese $8.2 \%$, Korean 5.8\%, Indian 4.2\%, Taiwanese 1.6\%, other Asian (referring \\ to Asians living outside China, Taiwan, India or Korea) $3.9 \%$ \\ ${ }^{\mathrm{b}}$ Calculated using the Cockcroft-Gault equation [31] \\ ${ }^{c}$ Patients with missing information are not shown \\ ${ }^{\mathrm{d}}$ Includes 144 with a UGT1A1*28 mutation, none with a UGT1A1*36 mutation and 2 with a UGT1A1*37 \\ mutation
}

non-smoker) who received nintedanib, the typical CL/F was $897 \mathrm{~L} / \mathrm{h}$, the $\mathrm{V}_{2} / \mathrm{F}$ in the central compartment at steady state was $465 \mathrm{~L}, k_{\mathrm{a}}$ was $0.0827 \mathrm{~h}^{-1}$, and ALAG was $0.417 \mathrm{~h}$. Evaluation of the population mean value of half-life suggests an elimination half-life of $0.36 \mathrm{~h}$ (calculated as $0.693 \times \mathrm{V}_{2} / \mathrm{CL}$ ), whereas the absorption half-life (calculated as $0.693 / \mathrm{ka}$ ) was $8.38 \mathrm{~h}$, suggesting flip-flop pharmacokinetic behavior [33], in which absorption becomes the rate-limiting metric of exposure. IIV in F1 expressed as coefficient of variation (CV) was $49.1 \%$ and IIV in $\mathrm{V}_{2} / \mathrm{F}$ and $k_{\mathrm{a}}$ were 119 and $32.4 \%$, respectively. IOV in $\mathrm{F} 1$ of small-to-moderate extent (CV $38.9 \%$ ) was detected. However, for most patients, pharmacokinetic samples were only available on two occasions and at two timepoints (one pre- and one post-dose). Considering that absorption might not follow perfect first-order kinetics, clear separation of IOV and residual variability (due to analytical precision of nintedanib measurements, imprecise sampling times, or model misspecifications) was challenging. Adding IOV to the nintedanib model only resulted in minor changes to the fixed-effect parameter estimates (including time varying covariate effects) and it was, therefore, not implemented. The $\eta$-shrinkages for IIV in $F 1, V_{2} / F$, and $k_{\mathrm{a}}$ were $13.9,60.0$, and $37.5 \%$, respectively. The overall $\varepsilon$-shrinkage was $12.7 \%$. The estimates for $k_{\mathrm{a}}$ and IIV in $k_{\mathrm{a}}$ were based on the Phase II trials [11, 23], as the absorption phase was considered to be captured better in these trials due to the higher number of available post-dose samples.

For the covariates age, body weight, ethnic origin, and smoking status, the model-predicted effects on the population mean nintedanib $\mathrm{AUC}_{\tau, \mathrm{ss}}$ are depicted in Fig. 1 and Table 4. Each of the covariates had a small-to-moderate influence on nintedanib exposure. Other than ethnic origin, the ratios of $\mathrm{AUC}_{\tau, \mathrm{ss}}$ for nintedanib for each factor were within the $80-125 \%$ range when varying those covariates individually within the observed extreme values (5th and 95th percentiles of baseline values for continuous covariates); exposure was increased by $33 \%$ in Chinese, Taiwanese, or Indian patients relative to a reference Caucasian patient (corrected for other covariate effects, particularly body weight).

Typical plasma profiles by subgroups of covariates with a significant influence on nintedanib plasma exposure are shown in Fig. 2. All of the median profiles based on individual covariate effects were within the $90 \%$ prediction interval profile for the typical patient.

\section{Final pharmacokinetic model for BIBF 1202}

The pharmacokinetic profile of BIBF 1202 was best described by a one-compartment model with first-order elimination (see Online Resource Fig. S1 for model structure). To account for formation of BIBF 1202 in the intestine or via hepatic first-pass effect, a first-order absorption with lag time $\left(\mathrm{ALAG}_{2}\right)$ was implemented as per nintedanib absorption. The relative bioavailability (F2) and the absorption-rate constant $\left(k_{\mathrm{a} 2}\right)$ were implemented as functions of nintedanib F1 and $k_{\mathrm{a}}$, respectively (substantial drop in OFV as compared to a model, where $\mathrm{F} 2$ and $k_{\mathrm{a} 2}$ were estimated independently of $\mathrm{F} 1$ and $k_{\mathrm{a}}$ ). This parametrization accounted for correlations between nintedanib and BIBF 1202 parameters and replaced the full variance-covariance matrix which was not applied due to the sequential modeling approach used. The rate of formation of BIBF $1202\left(k_{\mathrm{met}}\right)$ during nintedanib systemic elimination was accounted by defining it as a function of nintedanib clearance: $k_{\mathrm{met}}=\mathrm{CL} / \mathrm{V}_{2} * \mathrm{ffM}$, where $C L$ and $V_{2}$ denote the clearance and central volume of distribution of nintedanib respectively, and ffM denotes the proportion of the nintedanib elimination resulting in BIBF 1202 formation. Due to the lack of data for BIBF 1202 given as an intravenous (IV) infusion, several parameter estimates were not identifiable (ffM, BIBF 1202 apparent volume of distribution $\left[\mathrm{V}_{3} / \mathrm{F}\right]$ ) and were, therefore, fixed. 
Table 3 Parameter estimates from the final population pharmacokinetic model of nintedanib

\begin{tabular}{|c|c|c|}
\hline Parameter & Estimate $\left(\mathrm{RSE} \%^{\mathrm{a}}\right)$ & Bootstrap analysis, $95 \% \mathrm{CI}$ \\
\hline \multicolumn{3}{|l|}{ Structural model parameters (fixed effects) } \\
\hline $\mathrm{CL} / \mathrm{F}[\mathrm{L} / \mathrm{h}]\left(\theta_{\mathrm{CL}}\right)$ & $897(2.42)$ & $855-941$ \\
\hline $\mathrm{V}_{2} / \mathrm{F}[\mathrm{L}]\left(\theta_{\mathrm{V} 2}\right)$ & $465(10.7)$ & $376-569$ \\
\hline$k_{\mathrm{a}}\left[\mathrm{h}^{-1}\right]\left(\theta_{\mathrm{ka}}\right)$ & $0.0376(7.77)$ & $0.0323-0.0439$ \\
\hline ALAG $[\mathrm{h}]$ & $0.417(5.59)$ & $0.351-0.463$ \\
\hline $\mathrm{F} 1\left(\theta_{\mathrm{F} 1}\right)$ & $1.00^{\mathrm{b}}(-)$ & \\
\hline \multicolumn{3}{|l|}{ Covariate effects on $\mathrm{F} 1$} \\
\hline \multicolumn{3}{|l|}{$\theta_{\text {Ethnicity }}$} \\
\hline Caucasian/Black/other Asian origin & $1.00^{\mathrm{b}}(-)$ & - \\
\hline Indian/Chinese/Taiwanese origin & $1.33(5.21)$ & $1.19-1.47$ \\
\hline Korean origin & $0.781(6.53)$ & $0.690-0.893$ \\
\hline \multicolumn{3}{|l|}{$\theta_{\text {Smok }}$} \\
\hline Ex-or non-smoker & $1.00^{\mathrm{b}}(-)$ & - \\
\hline Current smoker & $0.794(4.46)$ & $0.725-0.864$ \\
\hline$\theta_{\text {Age }}$ & $0.00959(16.0)$ & $0.00635-0.0126$ \\
\hline \multicolumn{3}{|l|}{$\theta_{\text {Trial }}$} \\
\hline IPF Phase II [11] and LUME-Lung 1 [5] & $1.00^{\mathrm{b}}(-)$ & - \\
\hline NSCLC Phase II [23] and LUME-Lung 2 [6] & $1.30(3.77)$ & $1.21-1.39$ \\
\hline \multicolumn{3}{|l|}{ Covariate effects on CL/F } \\
\hline$\theta_{\mathrm{WT}}$ & $0.619(16.5)$ & $0.453-0.789$ \\
\hline \multicolumn{3}{|l|}{ Covariate effects on $k_{\mathrm{a}}$} \\
\hline \multicolumn{3}{|l|}{$\theta_{\text {Trial }}$} \\
\hline LUME-Lung 1 [5] and LUME-Lung 2 [6] & $1.00^{\mathrm{b}}(-)$ & \\
\hline NSCLC Phase II [23] and IPF Phase II [11] & $2.20(8.00)$ & $1.87-2.58$ \\
\hline \multicolumn{3}{|l|}{ Inter-individual variability } \\
\hline IIV in F1 [CV\%] & $49.1\left(6.64^{c}\right)$ & $45.7-52.1$ \\
\hline IIV in $k_{\mathrm{a}}$ for Phase II studies [CV\%] & $32.4\left(19.2^{c}\right)$ & $25.2-37.8$ \\
\hline IIV in $k_{\mathrm{a}}$ for Phase III studies [CV\%] & $53.8\left(33.8^{\mathrm{cd}}\right)$ & $0.00538-67.7^{\mathrm{d}}$ \\
\hline IIV in $\mathrm{V}_{2} / \mathrm{F}$ [CV\%] & $119\left(15.7^{c}\right)$ & $99.3-139$ \\
\hline \multicolumn{3}{|l|}{ Residual unexplained variability } \\
\hline Additive (SD) [nM; log scale] & $0.526\left(4.58^{\mathrm{c}}\right)$ & $0.504-0.553$ \\
\hline
\end{tabular}

$\mathrm{F} 1=1 \cdot \theta_{\text {Ethnicity }} \cdot\left(1+\theta_{\mathrm{Age}} \cdot(\mathrm{AGE}-62)\right) \cdot \theta_{\mathrm{Smok}} \cdot \theta_{\text {Trial }} \cdot \mathrm{e}^{\eta \mathrm{F} 1}$

$\mathrm{CL} / \mathrm{F}=\theta_{\mathrm{CL}} \cdot(\mathrm{WT} / 71.5)^{\theta \mathrm{WT}}$

$k_{\mathrm{a}}=\theta_{\mathrm{ka}} \cdot \theta_{\text {Trial }} \cdot \mathrm{e}^{\eta \mathrm{ka}}$

$\mathrm{V}_{2} / \mathrm{F}=\theta_{\mathrm{V} 2} \cdot \mathrm{e}^{\eta \mathrm{V} 2}$

$\mathrm{ALAG}=\theta_{\mathrm{ALAG}}$

$\theta$ fixed-effect parameter of interest, $A L A G$ absorption lag time of nintedanib, $C I$ confidence interval, $C L / F$ apparent total body clearance for nintedanib, $C V$ coefficient of variation, ECOG Eastern Cooperative Oncology Group, F1 relative bioavailability for nintedanib, IIV $(\eta)$ inter-individual variability, IPF idiopathic pulmonary fibrosis, $k_{a}$ first-order absorption-rate constant for nintedanib, $n M$ nanomolar (nintedanib concentration in $\mathrm{nM}=1.853 \times$ nintedanib concentration in $\mathrm{ng} / \mathrm{mL}$ ), NSCLC non-small-cell lung cancer, $R S E$ relative standard error, $V_{2} / F$ apparent volume of distribution for nintedanib, $S D$ Standard deviation

${ }^{a}$ The relative standard error as provided by NONMEM

${ }^{\mathrm{b}}$ Parameters were fixed to 0 or 1 as reference values

${ }^{c}$ Given on the variance scale

${ }^{\mathrm{d}}$ By excluding terminated bootstrap runs or runs with estimates near boundary, NONMEM and bootstrap results were congruent in estimating the imprecision measures (after exclusion of these runs, RSE\% and 95\% CI from bootstrapping were $37.0 \%$ and 22.6-68.0, respectively; otherwise the respective measures were $52.4 \%$ and $0.00538-67.7$ ) 
Fig. 1 Ratios (point estimates and $95 \%$ CIs based on bootstrap analysis) of nintedanib population mean exposure (AUC $\tau, \mathrm{ss}$ ) predicted by the final model for different covariates compared with a typical patient (Caucasian, non-smoker, age 62 years, body weight $71.5 \mathrm{~kg}$ ) receiving nintedanib treatment. The solid vertical line indicates the population mean for the typical patient, and the shaded area is the $90 \%$ prediction interval for inter-patient variability. The vertical dotted lines indicate the bioequivalence limits ( 80 $125 \%$ ). The 5th, 25th, 75th, and 95th percentiles of the baseline values observed in the analyzed population are shown for age and body weight

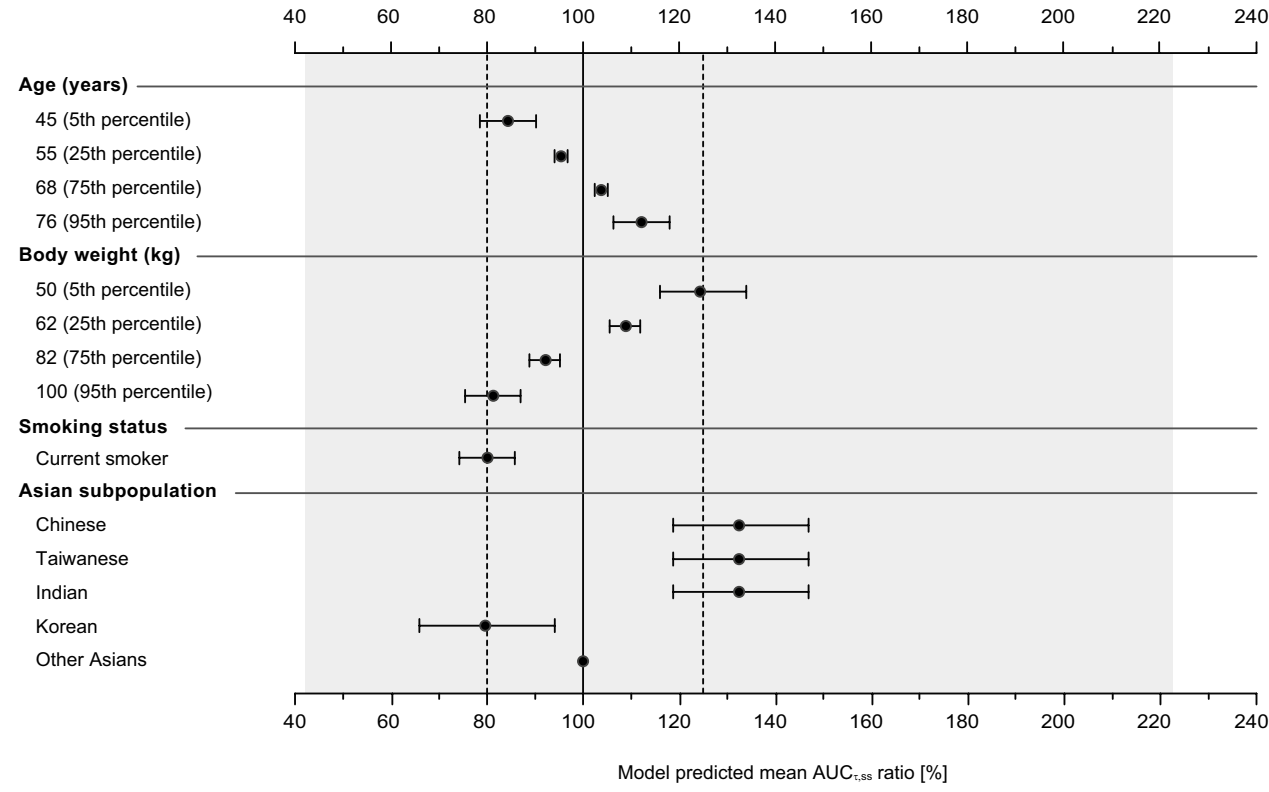

Model predicted mean $\mathrm{AUC}_{\tau, \mathrm{ss}}$ ratio [\%]

Point estimate from final population pharmacokinetic model and $95 \%$ confidence interval Reference patient: age 62 years, body weight $71.5 \mathrm{~kg}$, ex- or non-smoker, Caucasian Bioequivalence limits $(80-125 \%)$

$90 \%$ prediction interval for inter-patient variability

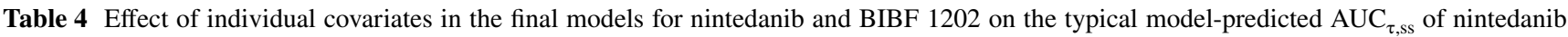
and BIBF 1202

\begin{tabular}{|c|c|c|c|}
\hline \multirow[t]{2}{*}{ Covariate } & \multirow[t]{2}{*}{ Reference patient } & \multicolumn{2}{|l|}{ Percentage change in $\mathrm{AUC}_{\tau, \mathrm{ss}}$} \\
\hline & & Nintedanib & BIBF 1202 \\
\hline Age & 62 years $^{\mathrm{a}}$ & $\begin{array}{l}45 \text { years }^{\mathrm{b}}: \downarrow 16 \% \\
76 \text { years }^{\mathrm{c}}: \uparrow 13 \%\end{array}$ & $\begin{array}{l}45 \text { years }^{\mathrm{b}}: \downarrow 16 \% \\
76 \text { years }^{\mathrm{c}}: \uparrow 13 \%\end{array}$ \\
\hline Smoking status & Ex- or non-smoker & Current smoker: $\downarrow 21 \%$ & Current smoker: $\downarrow 21 \%$ \\
\hline Body weight & $71.5 \mathrm{~kg}^{\mathrm{a}}$ & $\begin{array}{l}50 \mathrm{~kg}^{\mathrm{b}}: \uparrow 25 \% \\
100 \mathrm{~kg}^{\mathrm{c}}: \downarrow 19 \%\end{array}$ & $\begin{array}{l}50 \mathrm{~kg}^{\mathrm{b}}: \uparrow 32 \% \\
100 \mathrm{~kg}^{\mathrm{c}}: \downarrow 22 \%\end{array}$ \\
\hline Ethnic origin & Caucasian & $\begin{array}{l}\text { Chinese/Taiwanese/Indian: } \uparrow 33 \% \\
\text { Korean: } \downarrow 22 \%\end{array}$ & $\begin{array}{l}\text { Chinese/Taiwanese: } \uparrow 57 \% \\
\text { Indian: } \uparrow 141 \% \\
\text { Other Asians }{ }^{\mathrm{d}}: \uparrow 18 \% \\
\text { Korean: } \downarrow 8 \%\end{array}$ \\
\hline ECOG performance status & $\mathrm{ECOG} \geq 1$ & Not applicable & $\mathrm{ECOG}=0: \downarrow 12 \%$ \\
\hline Lactate dehydrogenase & $238 \mathrm{U} / \mathrm{L}^{\mathrm{a}}$ & Not applicable & $\begin{array}{l}141 \mathrm{U} / \mathrm{L}^{\mathrm{b}}: \downarrow 8 \% \\
576 \mathrm{U} / \mathrm{L}^{\mathrm{c}}: \uparrow 29 \%\end{array}$ \\
\hline
\end{tabular}

Reference patient is Caucasian, ex- or non-smoker, age 62 years, body weight $71.5 \mathrm{~kg}$, LDH level $238 \mathrm{U} / \mathrm{L}$, ECOG performance status $\geq 1$

Percentage change in $\mathrm{AUC}_{\tau, \mathrm{ss}}$ relative to reference patient was determined by varying values of the covariate of interest while keeping all other covariates constant. To illustrate effect size for continuous covariates, the 5 th and 95 th percentiles of respective baseline values were used

${ }^{a}$ Median of the baseline values observed in the analyzed population

${ }^{\mathrm{b}} 5$ th percentile

${ }^{\mathrm{c}} 95$ th percentile

${ }^{\mathrm{d}}$ Living outside China, Taiwan, India and Korea

The value for ffM was derived from pharmacokinetic data after IV administration of nintedanib [19] (Online Resource Table S4) and the ratio of $\mathrm{V}_{3} / \mathrm{F}-\mathrm{V}_{2} / \mathrm{F}$ was fixed based on information observed in rats [14]. Despite structural identifiability of $\mathrm{ALAG}_{2}$, this parameter was fixed to the value of nintedanib ALAG in the final model without a substantial 
Fig. 2 Median nintedanib plasma concentration-time profiles at steady state after multiple oral administration of nintedanib twice-daily (dose normalized) for different scenarios of covariate characteristics in relation with the median and $90 \%$ prediction interval for inter-patient variability of 2000 simulated profiles for a reference patient (Caucasian, non-smoker, age 62 years, body weight $71.5 \mathrm{~kg}$ ). For age and body weight effects, the 5 th and 95th percentiles of baseline values for the analyzed population are shown

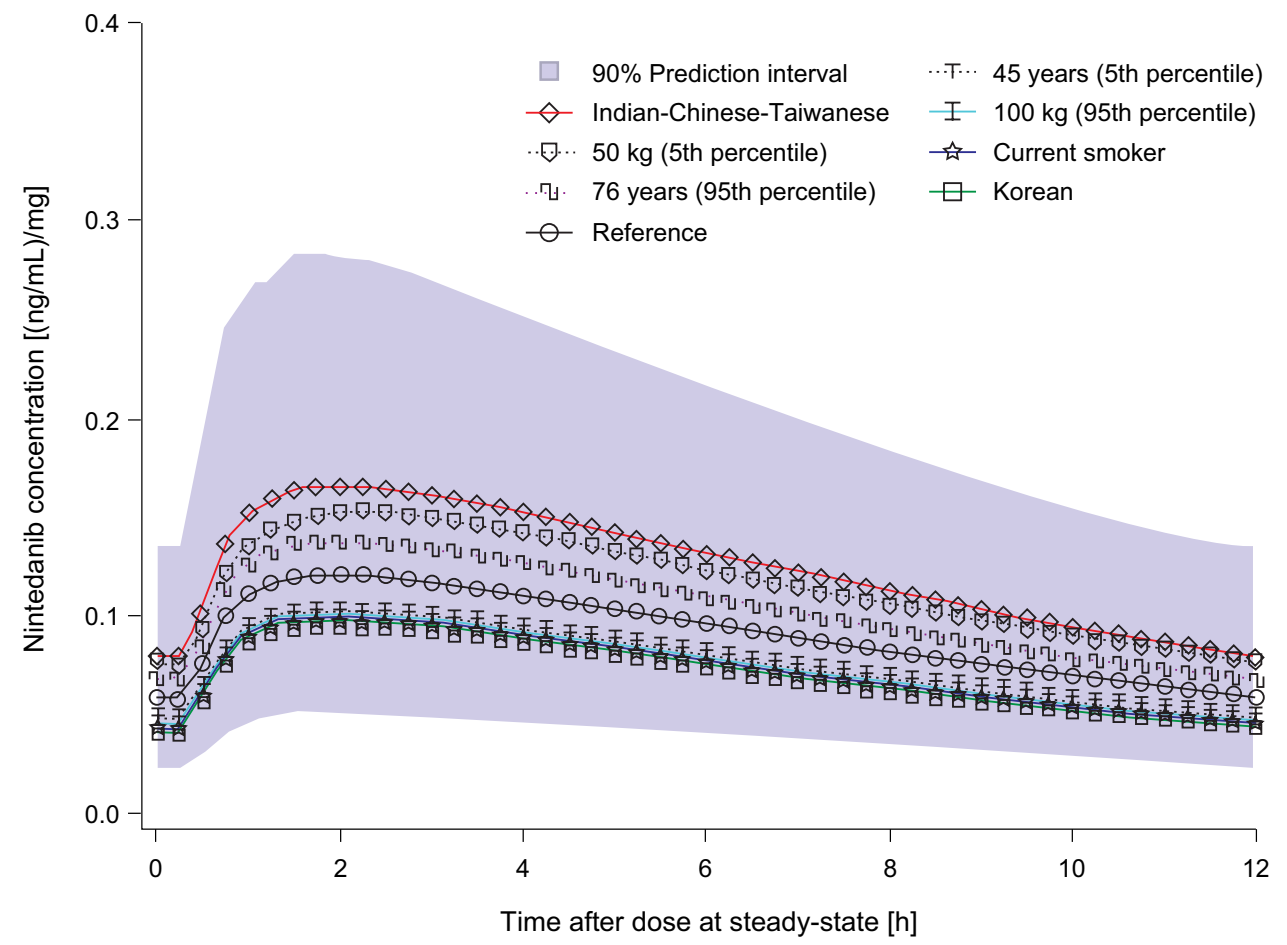

change in OFV $(\triangle \mathrm{OFV}=1.282)$, as besides the comparable estimates of $\mathrm{ALAG}_{2}$ and ALAG, the same absorption delay for both substances was considered plausible. In addition to the (inter-individual) variability of F2 induced by the variability in F1 (F2 being proportional to F1), additional IIV could be implemented for F2.

The final model parameter estimates for BIBF 1202 are shown in Online Resource Table S5.

For BIBF 1202, the same covariates were investigated as for nintedanib. The significant covariate effects for BIBF 1202 were either induced one-to-one by a change in nintedanib F1 (age and smoking status), were influenced by a change in nintedanib pharmacokinetics but with different effect sizes for BIBF 1202 (body weight, ethnic origin, trial effects), or were identified as BIBF 1202 specific effects (ECOG performance status, LDH, and NSCLC histology) (see Online Resource Table S6).

Table 4 displays the covariates with a significant effect on BIBF 1202 exposure $\left(\mathrm{AUC}_{\tau, \mathrm{ss}}\right)$. Besides ethnic origin, the covariates showed nearly identical effect sizes on BIBF 1202 exposure as compared to nintedanib. Additional covariates influencing BIBF 1202 exposure but not nintedanib exposure (i.e., ECOG performance status and LDH) showed only small-to-moderate changes $(<30 \%)$ in BIBF 1202 exposure. Parameter estimates for covariates not affecting BIBF 1202 exposure in terms of AUC (trial effects and NSCLC histology on $k_{\mathrm{a} 2}$ ) are shown in Online Resource Table S5.

Median steady-state plasma concentration-time profiles for patients with covariates at the extremes of the distribution were simulated (Online Resource Fig. S2). All of the median profiles based on individual covariate effects were within the $90 \%$ prediction interval profile for the typical patient.

\section{Simultaneous estimation of the final nintedanib and BIBF 1202 models}

Simultaneous estimation of all parameters for the final models of nintedanib and BIBF 1202 showed no major deviations of parameter estimates compared with the sequential fit (see Online Resource Table S7).

\section{Model evaluation and simulation}

The pcVPC for the base models of nintedanib and BIBF 1202 based on the total population (Online Resource Figs. S3 and S4) showed no major discrepancies between observed and simulated concentrations after multiple nintedanib administration. In addition to pcVPC, the corresponding pcQPC for the final model focusing on the predose (trough) and $2 \mathrm{~h}$ post-dose plasma concentration were performed. pcQPC results stratified by various covariates (Online Resource Figs. S5 and S6) show that nintedanib and BIBF 1202 exposure could be reasonably well predicted in the investigated subgroups. Only the steady-state trough levels of nintedanib at $24 \mathrm{~h}$ post-dose in patients with oncedaily dosing were under-predicted as apparent from the pcVPC in Online Resource Fig. S3. This can be explained 
by a high number of samples below the limit of quantification in this subgroup affecting the 5th percentile.

The non-parametric $95 \%$ confidence intervals (2.5th and 97.5th percentiles) from bootstrap analysis for the estimates of the final nintedanib model indicated that the parameters were precisely estimated (Table 3 ). The estimated CVs were generally less than $20 \%$ and were congruent with the relative standard error (RSE) estimated in NONMEM. An exception was the parameter IIV in $k_{\mathrm{a}}$ of the Phase III NSCLC trials, which could not be estimated adequately (CV $52.4 \%$ by bootstrap analysis versus RSE $33.8 \%$ in NONMEM).

\section{Discussion}

This population pharmacokinetic analysis investigated the pharmacokinetic profile of nintedanib and its main metabolite in the selected target patient populations of NSCLC and IPF. Data from 4 Phase II and III clinical trials were included. Overall, nintedanib exposure in the final model was reasonably well captured in predictive checks for the analyzed trial data and was, therefore, considered adequate for the prediction of nintedanib exposure overall and in subpopulations of covariate effects.

Nintedanib pharmacokinetic parameters were estimated by fitting a one-compartment structural model with an absorption lag, first-order input, and linear elimination to the plasma concentration versus time data. The slower rate of absorption $\left(k_{\mathrm{a}}\right)$ compared with the rate of elimination $\left(k_{\mathrm{e}}\right)$ suggests flip-flop pharmacokinetic behavior, where absorption is the rate-limiting process for elimination of nintedanib [33]. Though the trial data did not allow a clear differentiation between flip-flop and non-flip-flop pharmacokinetics, the flip-flop solution was pursued for the following reasons: (1) non-flip-flop-related parameter estimates could be obtained using different initial parameter values, but the OFV was consistently lower for the flip-flop solution. (2) When applying non-flip-flop kinetics in the final model, extreme estimates for $\mathrm{V}_{2}(10,700 \mathrm{~L})$ and $k_{\mathrm{a}}(1.64$ $1 / \mathrm{h}$ for Phase II trial data) were obtained, whereas minor changes for other fixed-effect parameters (including covariate effects) were obtained. (3) After IV administration, nintedanib exhibited multi-compartmental pharmacokinetics with a very steep decline in plasma concentrations after end of infusion; the terminal half-life was, however, essentially comparable to those after oral administration indicating a redistribution-driven terminal phase. Although the terminal half-life of nintedanib should be longer following oral administration compared with IV administration for true flip-flop pharmacokinetic behavior, the nintedanib plasma profiles after oral and IV administration, nevertheless, indicate that at least some distribution processes as well as the elimination from central compartment are faster than the absorption process [19]. As a one-compartment model cannot emulate the redistribution from a (deep) peripheral compartment, the flip-flop solution was considered to be more representative and appropriate for the chosen structural model (see also Online Resource Table S4 for pharmacokinetic parameters of a one-compartment model based on data from IV administration). (4) Finally, use of a one-compartment model was considered fit for the purpose of describing steady-state (mean) exposure and assessing impact of intrinsic and extrinsic factors and follows the most parsimonious model principle supported by the data. Measures needed to enable implementation of a two- or even three-compartment model would have added complexity, which was not considered to be outweighed by the prospective benefits. Overall, the chosen modeling approach was more empirical than mechanistic (e.g., the absorption-rate limitation is not conclusively supported by the IV data). However, this approach was considered suitable for identifying subpopulations with altered nintedanib exposure and provided a valuable basis for exposure-response analyses to guide labeling recommendations.

The significantly higher nintedanib exposure observed in most of the studied Asian subpopulations countries could not be explained by differences in body weight. At present, no hypothesis can explain the underlying mechanism for the small-to-moderate increase in exposure (up to 33\%) observed in the investigated Asian subpopulations. No Japanese patients were included in the trial data set.

The current analysis showed that although mild hepatic impairment, classified by elevated transaminase or bilirubin levels, showed a weak trend towards increased nintedanib exposure, it did not meet the significance criteria based on the forward inclusion-backward elimination procedure to be included in the final model. However, the limited number of patients with mild hepatic impairment $(9.7 \%$ of included patients) and the lack of information about underlying hepatic disease did not allow a robust assessment of this effect. Similarly, data did not allow robust assessment of moderate hepatic impairment and there were no data for patients with severe hepatic impairment. More recent data from a dedicated hepatic impairment trial in subjects with liver cirrhosis [34] indicate an approximate 2-fold and 8-fold increases in nintedanib exposure in patients with mild or moderate hepatic impairment, categorized according to the Child-Pugh classification [29], respectively. The current model showed that patients with liver metastases $(n=145)$ had no significant effect on nintedanib pharmacokinetics. Therefore, although liver metastases alone are not considered to influence the pharmacokinetics of nintedanib if associated with other indicators of hepatic impairment, the pharmacokinetics of nintedanib can be affected. Renal function, as determined by $\mathrm{CL}_{\mathrm{CR}}$, did not have a significant impact on nintedanib pharmacokinetics after accounting for 
body weight. This is consistent with renal excretion having a minor role in the elimination of nintedanib.

The influence of disease (IPF versus NSCLC) or concomitant chemotherapy (docetaxel or pemetrexed) on nintedanib pharmacokinetics was assessed by accounting for potential individual trial effects. Results showed that trial specific differences in nintedanib exposure and absorption could not be explained by underlying disease or concomitant therapy. Differences in bioavailability were unlikely to be related to concomitant chemotherapy, as patients in the monotherapy trials were estimated to have the same bioavailability as those in combination therapy trials. Similarly, disease indication effects were not obvious as bioavailability in the IPF trial was estimated to be the same as that in one NSCLC trial [5]. The estimated 2-fold higher absorptionrate constant in the Phase II trials versus the Phase III trials did also not suggest an indication or comedication effect and was likely due to the higher number of post-dose samples in these trials (up to two per visit versus one per visit). The sparse pharmacokinetics sampling in the Phase III clinical trials and their timing in relation with the chemotherapy infusion ( 1 week apart) together with the lack of plasma exposure for combination partners did not allow a proper drug-drug interaction (DDI) assessment. Dedicated Phase I DDI trials previously showed no significant pharmacokinetic interaction between nintedanib and either docetaxel or pemetrexed $[35,36]$. In this regard, this analysis provides confirmation of the Phase I DDI trial findings using longer term nintedanib treatment in the Phase III trials. Therefore, it was concluded that disease or concomitant chemotherapy did not show a systematic change in nintedanib exposure.

Despite a lower potency of BIBF 1202 exposure in in vivo assays, characterization of its pharmacokinetics, including interdependencies with the pharmacokinetics of nintedanib and identification of relevant intrinsic and extrinsic factors, was a secondary objective of this analysis, as a possible minor contribution of BIBF 1202 exposure to the overall effect in humans cannot be ruled out and effects of intrinsic and extrinsic factors could potentially cause a clinically relevant increase in BIBF 1202 exposure. Data from an earlier bioavailability trial [19] indicated a substantial formation of BIBF 1202 during first-pass metabolism. By assuming the same rate of systemic BIBF 1202 formation as estimated based on data from the previous study (fixed parameter for systemic formation rate), about $8 \%$ of BIBF 1202 in plasma was estimated to be formed systemically by the final current model.

In a dedicated absorption, distribution, metabolism, and elimination (ADME) study, the plasma concentration-time profiles of nintedanib and BIBF 1202 were similar, yielding a metabolic ratio close to 1 for AUC and $C_{\max }$ [16]. As the pharmacokinetics of BIBF 1202 are directly influenced by the nintedanib pharmacokinetics, the covariates influencing the parent compound indirectly also affected the pharmacokinetics of BIBF 1202. Most of the intrinsic and extrinsic factors affecting BIBF 1202 exposure did so in a manner similar to nintedanib, resulting in marginal changes in their corresponding metabolic ratio. Additional effects on BIBF 1202 exposure, particularly the higher differences in exposure for the ethnic subpopulations (e.g., a 141\% higher $\mathrm{AUC}_{\tau, \mathrm{ss}}$ for Indian patients and a $57 \%$ higher $\mathrm{AUC}_{\tau, \mathrm{ss}}$ for Chinese and Taiwanese patients as compared to Caucasian patients), are not expected to change this metabolic ratio by more than 2-2.5-folds. In view of the significantly lower in vivo potency of BIBF 1202 (9-to-several hundred-folds based on cellular assays, Boehringer Ingelheim unpublished data [14]), and the close relationship between nintedanib and BIBF 1202 exposure, conclusions from the nintedanib plasma pharmacokinetic data are considered relevant for interpretation of the clinical effects of nintedanib therapy, despite a possible minor contribution of BIBF 1202 exposure to the overall effect in humans.

\section{Conclusion}

In summary, the final model provides an adequate description of the pharmacokinetic profile of nintedanib and its main metabolite BIBF 1202 in the two target patient populations and can be used for simulations exploring covariate effects and for the development of exposure-response relationships. Patient gender, mild or moderate renal impairment, and mild hepatic impairment (classified by transaminase or bilirubin increase), underlying disease, treatment regimen, and the presence of liver metastases, had no significant effect on nintedanib exposure. The individual intrinsic and extrinsic covariates with a significant effect on nintedanib pharmacokinetics (body weight, age, smoking history, and ethnic origin) showed small-to-moderate proportional changes in exposure which individually did not exceed the observed inter-patient variability range. Similar pharmacokinetics and covariate effects were found for BIBF 1202. The results suggest that there is no need for a priori dose adjustment in the tested Asian patient subgroups (Chinese, Korean, Taiwanese, and other Asian), smokers, patients with very high or low body weight, or the young/ elderly. However, particularly, a combination of equally directed covariate effects could result in more pronounced changes in systemic exposure. Due to a potentially higher frequency of adverse events, close monitoring for tolerability is warranted for patients with elevated nintedanib exposure (e.g., due to Asian race, low body weight, high age, or combinations of these risk factors). 
Acknowledgements These studies were sponsored by Boehringer Ingelheim Pharma GmbH \& Co. KG, Germany. Boehringer Ingelheim was responsible for the design and conduct of all of the studies, and the collection and management of the data. The authors were responsible for the analysis and interpretation of the data and the preparation of the manuscript. The authors thank the medical staff of Boehringer Ingelheim responsible for each study, the investigators of each study, and the staff conducting the bioanalytical portion of the studies. Editorial support was provided by Paul Tanswell, with funding from Boehringer Ingelheim.

\section{Compliance with ethical standards}

Funding These studies were sponsored by Boehringer Ingelheim Pharma GmbH \& Co. KG, Germany.

Conflict of interest All authors are employees of Boehringer Ingelheim Pharma GmbH \& Co. KG. The authors declare that they have no other conflicts of interest directly related to the content of this analysis.

Ethical approval All procedures performed in studies involving human participants were in accordance with the ethical standards of the institutional and/or national research committee and with the 1964 Helsinki declaration and its later amendments or comparable ethical standards.

Informed consent Informed consent was obtained from all individual participants included in the studies.

Open Access This article is distributed under the terms of the Creative Commons Attribution 4.0 International License (http://creativecommons.org/licenses/by/4.0/), which permits unrestricted use, distribution, and reproduction in any medium, provided you give appropriate credit to the original author(s) and the source, provide a link to the Creative Commons license, and indicate if changes were made.

\section{References}

1. Hilberg F, Roth GJ, Krssak M, Kautschitsch S, Sommergruber W, Tontsch-Grunt U, Garin-Chesa P, Bader G, Zoephel A, Quant J, Heckel A, Rettig WJ (2008) BIBF 1120: triple angiokinase inhibitor with sustained receptor blockade and good antitumor efficacy. Cancer Res 68(12):4774-4782

2. Roth GJ, Binder R, Colbatzky F, Dallinger C, Schlenker-Herceg R, Hilberg F, Wollin SL, Kaiser R (2015) Nintedanib: from discovery to the clinic. J Med Chem 58(3):1053-1063

3. McCormack PL (2015) Nintedanib: first global approval. Drugs 75(1):129-139

4. Bouquet C, Lamande N, Brand M, Gasc JM, Jullienne B, Faure G, Griscelli F, Opolon P, Connault E, Perricaudet M, Corvol P (2006) Suppression of angiogenesis, tumor growth, and metastasis by adenovirus-mediated gene transfer of human angiotensinogen. Mol Ther 14(2): 175-182

5. Reck M, Kaiser R, Mellemgaard A, Douillard JY, Orlov S, Krzakowski M, von Pawel J, Gottfried M, Bondarenko I, Liao M, Gann CN, Barrueco J, Gaschler-Markefski B, Novello S (2014) Docetaxel plus nintedanib versus docetaxel plus placebo in patients with previously treated non-small-cell lung cancer (LUME-Lung 1): a phase 3, double-blind, randomised controlled trial. Lancet Oncol 15(2):143-155
6. Hanna NH, Kaiser R, Sullivan RN, Aren OR, Ahn MJ, Tiangco B, Voccia I, Pawel JV, Kovcin V, Agulnik J, Gaschler-Markefski B, Barrueco J, Sikken P, Schloss C, Kim JH (2016) Nintedanib plus pemetrexed versus placebo plus pemetrexed in patients with relapsed or refractory, advanced non-small cell lung cancer (LUME-Lung 2): A randomized, double-blind, phase III trial. Lung Cancer 102:65-73

7. European Medicines Agency (2014) Committee for Medicinal Products for Human Use (CHMP) Assessment Report for Vargatef (nintedanib), 25 September 2014. http://www.ema.europa. eu/docs/en_GB/document_library/EPAR_-_Public_assessment_report/human/002569/WC500179972.pdf. Accessed 29 Aug 2017

8. Selman M, King TE, Pardo A (2001) Idiopathic pulmonary fibrosis: prevailing and evolving hypotheses about its pathogenesis and implications for therapy. Ann Intern Med 134(2):136-151

9. Wollin L, Maillet I, Quesniaux V, Holweg A, Ryffel B (2014) Antifibrotic and anti-inflammatory activity of the tyrosine kinase inhibitor nintedanib in experimental models of lung fibrosis. $\mathbf{J}$ Pharmacol Exp Ther 349(2):209-220

10. Wollin L, Wex E, Pautsch A, Schnapp G, Hostettler KE, Stowasser S, Kolb M (2015) Mode of action of nintedanib in the treatment of idiopathic pulmonary fibrosis. Eur Respir J 45(5):1434-1445

11. Richeldi L, Costabel U, Selman M, Kim DS, Hansell DM, Nicholson AG, Brown KK, Flaherty KR, Noble PW, Raghu G, Brun M, Gupta A, Juhel N, Klüglich M, du Bois RM (2011) Efficacy of a tyrosine kinase inhibitor in idiopathic pulmonary fibrosis. N Engl J Med 365(12):1079-1087

12. Richeldi L, du Bois RM, Raghu G, Azuma A, Brown KK, Costabel U, Cottin V, Flaherty KR, Hansell DM, Inoue Y, Kim DS, Kolb M, Nicholson AG, Noble PW, Selman M, Taniguchi H, Brun M, Le MF, Girard M, Stowasser S, Schlenker-Herceg R, Disse B, Collard HR, INPULSIS Trial Investigators (2014) Efficacy and safety of nintedanib in idiopathic pulmonary fibrosis. N Engl J Med 370 (22):2071-2082

13. Boehringer Ingelheim (2017) OFEV® (nintedanib) capsules, for oral use; Prescribing information, revised: 08/2017. http://docs. boehringer-ingelheim.com/Prescribing\%20Information/PIs/Ofev/ ofev.pdf. Accessed 30 Aug 2017

14. European Medicines Agency (2014) Committee for Medicinal Products for Human Use (CHMP) Assessment Report for Ofev(nintedanib), 20 November 2014. http://www.ema.europa. eu/docs/en_GB/document_library/EPAR_-_Public_assessment_ report/human/003821/WC500182476.pdf. Accessed 12 May 2017

15. Mross K, Stefanic M, Gmehling D, Frost A, Baas F, Unger C, Strecker R, Henning J, Gaschler-Markefski B, Stopfer P, de Rossi L, Kaiser R (2010) Phase I study of the angiogenesis inhibitor BIBF 1120 in patients with advanced solid tumors. Clin Cancer Res 16(1):311-319

16. Stopfer P, Rathgen K, Bischoff D, Ludtke S, Marzin K, Kaiser R, Wagner K, Ebner T (2011) Pharmacokinetics and metabolism of BIBF 1120 after oral dosing to healthy male volunteers. Xenobiotica 41(4):297-311

17. Boehringer Ingelheim (2006) Safety and pharmacokinetics/bioavailability of a single dose of $150 \mathrm{mg}$ BIBF 1120 administered as soft gelatine capsules with and without food to healthy male volunteers in an open, randomised, intra-individual crossover comparison design, Phase I trial. Trial No. 1199.17. http://www. trials.boehringer-ingelheim.com/public/trial_results_documents/1199/1199.17_U06-1411-02.pdf. Accessed 12 May 2017

18. Ogura T, Taniguchi $\bar{H}$, Azuma A, Inoue Y, Kondoh Y, Hasegawa Y, Bando M, Abe S, Mochizuki Y, Chida K, Kluglich M, Fujimoto T, Okazaki K, Tadayasu Y, Sakamoto W, Sugiyama Y (2015) Safety and pharmacokinetics of nintedanib and pirfenidone in idiopathic pulmonary fibrosis. Eur Respir J 45(5):1382-1392 
19. Dallinger C, Trommeshauser D, Marzin K, Liesener A, Kaiser R, Stopfer P (2016) Pharmacokinetic properties of nintedanib in healthy volunteers and patients with advanced cancer. J Clin Pharmacol 56(11):1387-1394

20. Eisen T, Shparyk Y, Macleod N, Jones R, Wallenstein G, Temple G, Khder Y, Dallinger C, Studeny M, Loembe AB, Bondarenko I (2013) Effect of small angiokinase inhibitor nintedanib (BIBF 1120) on QT interval in patients with previously untreated, advanced renal cell cancer in an open-label, phase II study. Invest New Drugs 31(5):1283-1293

21. Boehringer Ingelheim (2013) Relative bioavailability of nintedanib given alone and in combination with ketoconazole at steady state in healthy male volunteers (an open-label, randomised, two-way cross-over clinical Phase I study). Trial No. 1199.161. http://www.trials.boehringer-ingelheim.com/public/ trial_results_documents/1199/1199.161_U13-1925-01-DS_ DR.pdf. Accessed 12 May 2017

22. Boehringer Ingelheim (2013) Relative bioavailability of a single oral dose of nintedanib given alone and in combination with multiple oral doses of rifampicin in healthy male volunteers (an open-label, two-period, fixed-sequence clinical Phase I trial). Trial No. 1199.162. http://www.trials.boehringer-ingelheim. com/public/trial_results_documents/1199/1199.162_U13-147801-DS_DR.pdf. Accessed 12 May 2017

23. Reck M, Kaiser R, Eschbach C, Stefanic M, Love J, Gatzemeier U, Stopfer P, von Pawel J (2011) A phase II double-blind study to investigate efficacy and safety of two doses of the triple angiokinase inhibitor BIBF 1120 in patients with relapsed advanced nonsmall-cell lung cancer. Ann Oncol 22(6):1374-1381

24. Lindbom L, Ribbing J, Jonsson EN (2004) Perl-speaks-NONMEM (PsN) - a Perl module for NONMEM related programming. Comput Methods Programs Biomed 75(2):85-94

25. Lindbom L, Pihlgren P, Jonsson EN (2005) PsN-Toolkit-a collection of computer intensive statistical methods for non-linear mixed effect modeling using NONMEM. Comput Methods Programs Biomed 79(3):241-257

26. Yardley DA (2009) Proactive management of adverse events maintains the clinical benefit of ixabepilone. Oncologist 14(5):448-455

27. Bristol-Myers Squibb (2016) Ixempra Kit (ixabepilone) for injection, for intravenous infusion only. Prescribing information, revised: 01/2016. http://ixempra.com/downloads/full_pi.pdf. Accessed 12 May 2017

28. LoRusso PM, Venkatakrishnan K, Ramanathan RK, Sarantopoulos J, Mulkerin D, Shibata SI, Hamilton A, Dowlati A, Mani S, Rudek MA, Takimoto CH, Neuwirth R, Esseltine DL, Ivy P (2012) Pharmacokinetics and safety of bortezomib in patients with advanced malignancies and varying degrees of liver dysfunction: phase I NCI Organ Dysfunction Working Group Study NCI-6432. Clin Cancer Res 18(10):2954-2963

29. Food and Administration Drug (2003) Guidance for Industry. Pharmacokinetics in patients with impaired hepatic function: Study design, data analysis, and impact on dosing and labeling. https://www.fda.gov/downloads/Drugs/GuidanceComplianceRegulatoryInformation/Guidances/ucm072123.pdf. Accessed 12 May 2017

30. Synold TW, Takimoto CH, Doroshow JH, Gandara D, Mani S, Remick SC, Mulkerin DL, Hamilton A, Sharma S, Ramanathan RK, Lenz HJ, Graham M, Longmate J, Kaufman BM, Ivy P (2007) Dose-escalating and pharmacologic study of oxaliplatin in adult cancer patients with impaired hepatic function: a National Cancer Institute Organ Dysfunction Working Group study. Clin Cancer Res 13(12):3660-3666

31. Cockcroft DW, Gault MH (1976) Prediction of creatinine clearance from serum creatinine. Nephron 16(1):31-41

32. Bergstrand M, Hooker AC, Wallin JE, Karlsson MO (2011) Prediction-corrected visual predictive checks for diagnosing nonlinear mixed-effects models. AAPS J 13(2):143-151

33. Yáñez JA, Remsberg CM, Sayre CL, Forrest ML, Davies NM (2011) Flip-flop pharmacokinetics-delivering a reversal of disposition: challenges and opportunities during drug development. Ther Deliv 2(5):643-672

34. Boehringer Ingelheim (2015) Pharmacokinetics, safety and tolerability of nintedanib single oral dose in male and female patients with different degrees of hepatic impairment (Child-Pugh classification $\mathrm{A}$ and $\mathrm{B}$ ) as compared with nintedanib administration to male and female healthy subjects (a non-blinded, parallel group study of Phase I), Phase I trial. Trial No. 1199.200. http:// www.trials.boehringer-ingelheim.com/public/trial_results_documents/1199/1199.200_c03149997-01_DR.pdf. Accessed 12 May 2017

35. Ellis PM, Kaiser R, Zhao Y, Stopfer P, Gyorffy S, Hanna N (2010) Phase I open-label study of continuous treatment with BIBF 1120, a triple angiokinase inhibitor, and pemetrexed in pretreated non-small cell lung cancer patients. Clin Cancer Res 16(10):2881-2889

36. Okamoto I, Miyazaki M, Takeda M, Terashima M, Azuma K, Hayashi H, Kaneda H, Kurata T, Tsurutani J, Seto T, Hirai F, Konishi K, Sarashina A, Yagi N, Kaiser R, Nakagawa K (2015) Tolerability of nintedanib (BIBF 1120) in combination with docetaxel: a phase 1 study in Japanese patients with previously treated non-small-cell lung cancer. J Thorac Oncol 10(2):346-352 